Pacific

Journal of

Mathematics

RICCI CURVATURE AND MINIMAL SUBMANIFOLDS

Thomas Hasanis and Theodoros Vlachos

Volume $197 \quad$ No. 1

January 2001 


\title{
RICCI CURVATURE AND MINIMAL SUBMANIFOLDS
}

\author{
Thomas Hasanis and Theodoros Vlachos
}

\begin{abstract}
The aim of this paper is to find necessary conditions for a given complete Riemannian manifold to be realizable as a minimal submanifold of a unit sphere.
\end{abstract}

\section{Introduction.}

The general question that served as the starting point for this paper was to find necessary conditions on those Riemannian metrics that arise as the induced metrics on minimal hypersurfaces or submanifolds of hyperspheres of a Euclidean space.

There is an abundance of complete minimal hypersurfaces in the unit hypersphere $S^{n+1}$. We recall some well known examples. Let $S^{m}(r)=\{x \in$ $\left.R^{n+1},|x|=r\right\}, S^{n-m}(s)=\left\{y \in R^{n-m+1},|y|=s\right\}$, where $r$ and $s$ are positive numbers with $r^{2}+s^{2}=1$; then $S^{m}(r) \times S^{n-m}(s)=\left\{(x, y) \in R^{n+2}, x \in\right.$ $\left.S^{m}(r), y \in S^{n-m}(s)\right\}$ is a hypersurface of the unit hypersphere in $R^{n+2}$. As is well known, this hypersurface has two distinct constant principal curvatures: One is $s / r$ of multiplicity $m$, the other is $-r / s$ of multiplicity $n-m$. This hypersurface is called a Clifford hypersurface. Moreover, it is minimal only in the case $r=\sqrt{m / n}, s=\sqrt{(n-m) / n}$ and is called a Clifford minimal hypersurface. Otsuki [11] proved that if $M^{n}$ is a compact minimal hypersurface in $S^{n+1}$ with two distinct principal curvatures of multiplicity greater than 1 , then $M^{n}$ is a Clifford minimal hypersurface $S^{m}(\sqrt{m / n}) \times S^{n-m}(\sqrt{(n-m) / n}), 1<m<n-1$. Furthermore, Otsuki constructed infinitely many compact minimal hypersurfaces, other than $S^{1}(\sqrt{1 / n}) \times S^{n-1}(\sqrt{(n-1) / n})$, with two distinct principal curvatures and one of them be simple, which are not congruent to each other in $S^{n+1}$. Using the method of equivariant Differential Geometry, families of infinitely many nonequitorial minimal embeddings of the $n$-sphere were constructed in $S^{n+1}$ for the dimensions $n=3,4,5,6,7,9,11$ and 13 by Hsiang and Sterling in [7]. Moreover, the same method will also produce at least one nonequitorial in all even dimensional spheres $([\mathbf{1 6}])$. These results settled the so called spherical Bernstein problem posed by S.S. Chern.

Let $M^{n}$ be an $n$-dimensional smooth and oriented Riemannian manifold, and $f: M^{n} \rightarrow S^{n+1}$ an isometric immersion of $M^{n}$ into the unit hypersphere $S^{n+1}$ of $R^{n+2}$. The unit normal vectorfield of $f$ in $S^{n+1}$ induces a map 
$\nu: M^{n} \rightarrow S^{n+1}$, the Gauss map of the immersion $f$. If we pull back onto $M^{n}$ through $\nu$ the standard metric of $S^{n+1}$, we obtain a 2-covariant tensorfield on $M^{n}$, which is a new metric on $M^{n}$ provided that the shape operator of $f$ is non-singular everywhere. Using this metric, the first author and Koutroufiotis in [6] proved: Let $M^{3}$ be a complete 3-dimensional oriented Riemannian manifold, and $f: M^{3} \rightarrow S^{4}$ an isometric minimal immersion; then the Ricci curvature Ric satisfies supRic $\geq 3 / 2$. If $M^{3}$ is compact, then supRic $>3 / 2$, unless $f\left(M^{3}\right)=S^{1}(\sqrt{1 / 3}) \times S^{2}(\sqrt{2 / 3})$.

The Clifford minimal hypersurface $S^{m}(\sqrt{m / n}) \times S^{n-m}(\sqrt{(n-m) / n}), 1 \leq$ $m \leq n-1$ is compact and its Ricci curvature varies between $n(m-1) / m$ and $n(n-m-1) /(n-m)$. So, the Ricci curvature of any Clifford minimal hypersurface satisfies infRic $\leq d_{n}$ and supRic $\geq c_{n}$, where $c_{n}=d_{n}=n-2$, if $n$ is even and $d_{n}=n(n-3) /(n-1), c_{n}=n(n-1) /(n+1)$, if $n$ is odd. These are the clues for our main results:

Theorem A. Let $M^{n}$ be a complete, oriented $n$-dimensional Riemannian manifold and $f: M^{n} \rightarrow S^{n+1}$ an isometric minimal immersion. Then supRic $\geq n-2$. Moreover,

(i) If $n=2 m$, then supRic $=n-2$ if and only if $f\left(M^{n}\right)=S^{m}(\sqrt{1 / 2}) \times$ $S^{m}(\sqrt{1 / 2})$.

(ii) If $n=2 m+1$, then supRic $>n-2$, unless the universal cover of $M^{n}$ is homeomorphic to $S^{n}$.

Theorem B. Let $M^{n}$ be a compact, oriented odd-dimensional minimal submanifold of the unit sphere $S^{n+k}$. Assume that the Ricci curvature satisfies Ric $>n(n-3) /(n-1)$. Then

(i) if $n \neq 3$, then $M^{n}$ is homeomorphic to $S^{n}$;

(ii) if $n=3$, then $M^{n}$ is topologically a space form of positive sectional curvature.

The following corollary is an immediate consequence of Theorem B.

Corollary. Let $M^{n}$ be a compact, oriented odd-dimensional Riemannian manifold which is not homeomorphic to $S^{n}$ if $n \neq 3$, or to a spherical space form if $n=3$. A necessary condition for $M^{n}$ to be realized as a minimal immersed submanifold of a unit hypersphere is that infRic $\leq n(n-3) /(n-$ $1)$.

Remark 1. In an attempt to extend Efimov's inequality to hypersurfaces in $S^{n+1}$, Smyth [13] proved the following: Let $M^{n}$ be a complete, oriented $n$-dimensional Riemannian manifold with sectional curvatures bounded from below and $f: M^{n} \rightarrow S^{n+1}$ an isometric immersion. If $M^{n}$ is not diffeomorphic to $R^{n}$ and not homeomorphic to a finite quotient of an odd-dimensional sphere, then supRic $\geq n-2$. 
Remark 2. The totally geodesic great spheres in $S^{n+1}$ satisfy the assumption of Theorem B on the Ricci curvature. However, the class of minimal submanifolds in a hypersphere with Ricci curvature Ric $>n(n-3) /(n-1)$ is not trivial. In fact, Do Carmo and Warner [2] proved that for each positive integer $s$ there exists an isometric minimal immersion $\psi_{s}$ of the 3 -dimensional sphere $S_{k(s)}^{3}$, with constant sectional curvature $k(s)=3 / s(s+2)$, into the unit hypersphere $S^{m(s)}$, where $m(s)=s(s+2)$. It is obvious that $\psi_{s}$ satisfies the assumption of Theorem B. Moreover, the assumption that $n$ is odd is essential. The Clifford minimal hypersurface $S^{m}(\sqrt{1 / 2}) \times S^{m}(\sqrt{1 / 2})$ is an example of a compact even dimensional minimal hypersurface in $S^{2 m+1}$, which is not homeomorphic to a sphere and satisfies the assumption on Ricci curvature. Leung [9] has proved that a compact, oriented $n$-dimensional minimal submanifolds of the unit hypersphere is homeomorphic to a sphere if the square length $S$ of the second fundamental form satisfies $S<n$. Theorem B is not a consequence of Leung's result since there exist minimal submanifolds of the unit hypersphere with $S>n$ which are homeomorphic to a sphere, for example $\psi_{s}, s \geq 2$.

The paper is organized as follows: Section 2, is devoted to some notations and preliminaries. In Section 3, we give the proofs of the main results. The paper ends up with some concluding remarks and questions.

\section{Preliminaries.}

Let $\left(M^{n},\langle\rangle,\right)$ be an $n$-dimensional smooth, oriented Riemannian manifold and $f: M^{n} \rightarrow S^{n+k}$ an isometric immersion of codimension $k$ into the unit hypersphere $S^{n+k}$ of $R^{n+k+1}$. Denote the standard connection of $S^{n+k}$ by $\vec{\nabla}$, the Riemannian connection of $M^{n}$ by $\nabla$, and the second fundamental form of the immersion by $B$. For tangent vectors $X$ and $Y$ of $M^{n}$, we have the Gauss formula

$$
\bar{\nabla}_{X} Y=\nabla_{X} Y+B(X, Y)
$$

and the Weingarten formula

$$
\bar{\nabla}_{X} e=-A_{e} X+D_{X} e,
$$

where the $(1,1)$ tensorfield $A_{e}$ is the shape operator associated with a normal vectorfield $e$, and $D$ is the connection in the normal bundle of $M^{n}$. It is well known that $\left\langle A_{e} X, Y\right\rangle=\langle B(X, Y), e\rangle$. Let $\left\{e_{1}, \ldots, e_{k}\right\}$ be a local orthonormal frame field in the normal bundle of $M^{n}$. For any unit tangent vector $X$ of $M^{n}$ the Ricci curvature $\operatorname{Ric}(X)$ in the direction of $X$ is given by

$$
\operatorname{Ric}(X)=n-1+\sum_{\alpha=1}^{k}\left(\operatorname{tr} A_{\alpha}\right)\left\langle A_{\alpha} X, X\right\rangle-\sum_{\alpha=1}^{k}\left|A_{\alpha} X\right|^{2},
$$


where $A_{\alpha}$ denotes the shape operator associated with $e_{\alpha}$. In particular, for a minimal immersion we have

$$
\operatorname{Ric}(X)=n-1-\sum_{\alpha=1}^{k}\left|A_{\alpha} X\right|^{2} .
$$

It is well known that if the normal connection $D$ is flat, then at each point there exists an orthonormal basis of the tangent space which simultaneously diagonalizes all shape operators. Moreover, in this case the Ricci curvature attains its absolute extrema at principal directions.

We restrict ourselves to immersions of codimension $k=1$. Let $\left(M^{n},\langle\rangle,\right)$ be an $n$-dimensional smooth and oriented Riemannian manifold, and $f$ : $M^{n} \rightarrow S^{n+1}$ an isometric immersion of $M^{n}$ into the unit hypersphere $S^{n+1}$. Denote by $N$ the unit normal vectorfield of $f$ in $S^{n+1}$ with associated shape operator $A$. We denote by $\Lambda$ the so called principal curvature set which is the set of values assumed by the principal curvatures and write $\bar{\Lambda}$ for the closure of $\Lambda$ in $\bar{R}$ the extended real numbers. We set $\Lambda^{ \pm}=\Lambda \cap R^{ \pm}$.

The map $f_{t}=\cos t f+\sin t N$ is an immersion if $\cot t=k \notin \Lambda$ and is in fact an isometric immersion if we endow $M^{n}$ with the pull back metric $\langle,\rangle_{t}$ via $f_{t}$. Its shape operator $A_{t}$ with respect to the unit normal vector $N_{t}=\cos t N-\sin t f$ is given by

$$
A_{t}=(k A+I)(k I-A)^{-1} .
$$

Obviously, if the Riemannian manifold $\left(M^{n},\langle\rangle,\right)$ is complete, then $\left(M^{n},\langle,\rangle_{t}\right)$ is complete if $k \notin \bar{\Lambda}$. For $t=\pi / 2$, the immersion $f_{\pi / 2}$ is the Gauss mapping with unit normal vectorfield $-f$ and associated shape operator $-A^{-1}$. If the principal curvatures of $f$ are $\lambda_{i}, i=1, \ldots, n$, then the principal curvatures of $f_{t}$ are

$$
\lambda_{i}(t)=\frac{k \lambda_{i}+1}{k-\lambda_{i}} .
$$

The principal curvature set $\Lambda_{t}$ of $f_{t}$ is related to the principal curvature set $\Lambda$ of $f$ by the Möbius transformation $\rho(x)=(k x+1) /(k-x)$. It is obvious that $\Lambda$ and $\Lambda_{t}$ have the same number of connected components.

The following auxiliary result is crucial for the proof of the Theorem A and is a combination of some lemmas given in $[\mathbf{1 3}, \mathbf{1 4}]$. For the sake of completeness we give a short proof of it.

Lemma 2.1. Let $M^{n}$ be a compact oriented Riemannian manifold and $f$ : $M^{n} \rightarrow S^{n+1}$ an isometric immersion with principal curvature set $\Lambda$. If $\Lambda^{ \pm}$ are both non-empty and $\inf \Lambda^{+} \sup \Lambda^{-}<-1$, then $0 \in \Lambda$.

Proof. Assume that $0 \notin \Lambda$. Choose a positive number $t>0$, such that $\inf \Lambda^{+}>k>-1 / \sup \Lambda^{-}$, where $k=\cos t / \sin t$. Obviously, the map $f_{t+\pi / 2}$ is an isometric immersion with principal curvatures $\lambda_{i}(t+\pi / 2)=\left(\lambda_{i}-\right.$ 
$k) /\left(1+k \lambda_{i}\right)$, as it follows from $(2.2)$, since $\cot (t+\pi / 2)=-1 / k$. We easily deduce that all principal curvatures of $f_{t+\pi / 2}$ are positive, that is, its shape operator is positive definite everywhere. It follows from Do Carmo-Warner [3] that $M^{n}$ is diffeomorphic to $S^{n}$ and $f_{t+\pi / 2}$ embeds it as the boundary of a convex domain in an open hemisphere. Moreover, $\Lambda_{t+\pi / 2}$ is connected and so $\Lambda$ must be connected. This contradicts our assumption that $0 \notin \Lambda$. So $0 \in \Lambda$.

\section{Proofs of the results.}

Proof of Theorem A. Arguing indirectly, we assume that supRic $=a<n-2$. Bearing in mind (2.1), for each principal curvature $\lambda \in \Lambda$ we have

$$
|\lambda| \geq \sqrt{n-1-a} .
$$

The map $\tilde{f}=f_{t}$ for $t=\pi / 2$ is an immersion. We denote by $\langle\tilde{,}\rangle$ the induced metric on $M^{n}$ via $\tilde{f}$. Then $\tilde{f}$ becomes an isometric immersion with shape operator $\tilde{A}=-A^{-1}$. Since $0 \notin \bar{\Lambda}$, the Riemannian manifold $\left.\left(M^{n}, \tilde{\zeta}_{,}\right\rangle\right)$is complete. The sectional curvature $\tilde{K}$ of $\left(M^{n}, \tilde{\langle,\rangle}\right)$ for the plane spanned by the orthogonal principal directions $e_{1}, e_{2}$ is

$$
\tilde{K}\left(e_{1} \wedge e_{2}\right)=1+\frac{1}{\lambda \mu},
$$

where $\lambda, \mu$ are the corresponding principal curvatures. Using (3.1), we easily see that

$$
\tilde{K} \geq \frac{n-2-a}{n-1-a} .
$$

By the Bonnet-Myers' theorem, we deduce that $M^{n}$ is compact. This implies that there is a point $P \in M^{n}$ and a principal curvature $\lambda(P)$ such that $|\lambda(P)|=\sqrt{n-1-a}$. Then, either inf $\Lambda^{+}=\sqrt{n-1-a}$, or $\sup \Lambda^{-}=-\sqrt{n-1-a}$. Moreover, from (3.1) we get $\sup \Lambda^{-} \leq-\sqrt{n-1-a}$ and $\inf \Lambda^{+} \geq \sqrt{n-1-a}$. Since $a<n-2$, in any case we obtain

$$
\inf \Lambda^{+} \sup \Lambda^{-} \leq-(n-1-a)<-1 .
$$

Appealing to Lemma 2.1, we infer that $0 \in \Lambda$, which contradicts (3.1). Hence we have proved that supRic $\geq n-2$.

Now assume that supRic $=n-2$. Then for each $\lambda \in \Lambda$ we have

$$
|\lambda| \geq 1 \text {. }
$$

We consider again the map $\tilde{f}=f_{t}$ for $t=\pi / 2$. The map $\tilde{f}$ is an immersion with shape operator $\tilde{A}=-A^{-1}$ and induced metric $\tilde{\langle,\rangle}$. Moreover, (3.2) implies that $\left(M^{n}, \tilde{\langle,\rangle}\right)$ is complete. Denote by $\lambda_{i}, i=1, \ldots, n$, the principal 
curvatures of $f$ and by $e_{i}, i=1, \ldots, n$, the corresponding principal directions. The sectional curvature $\tilde{K}$ of $\left(M^{n}, \tilde{\langle,\rangle}\right)$ for the plane spanned by the orthogonal principal directions $e_{i}, e_{j},(i \neq j)$ is

$$
\tilde{K}\left(e_{i} \wedge e_{j}\right)=1+\frac{1}{\lambda_{i} \lambda_{j}} .
$$

Case 1. Assume that $n=2$. In this case the map $\tilde{f}$ is an isometric minimal immersion of the complete Riemannian manifold $\left(M^{2},\langle\tilde{,}\rangle\right)$ with shape operator $\tilde{A}=-A^{-1}$ and Gaussian curvature $\tilde{K} \geq 0$, as it follows from (3.3). Hence $M^{2}$ is either compact or parabolic. We proceed now in a standard way. Using Simon's formula, we find that $\tilde{\Delta} \operatorname{tr} A^{-2} \geq 0$, where $\tilde{\Delta}$ is the Laplace operator of $\left.\left(M^{2}, \tilde{\zeta,}\right\rangle\right)$; therefore, $\operatorname{tr} A^{-2}=$ const., because $\operatorname{tr} A^{-2}$ is bounded from above. Thus, $\lambda_{1}=-\lambda_{2}=1$ and $f\left(M^{2}\right)=S^{1}(\sqrt{1 / 2}) \times$ $S^{1}(\sqrt{1 / 2})$.

Case 2. Assume that $n \geq 3$. Because of (3.2), we may suppose, after an eventual change of orientation, that

$$
\lambda_{1} \geq, \ldots, \geq \lambda_{k} \geq 1>-1 \geq \lambda_{k+1} \geq, \ldots, \geq \lambda_{n},
$$

with $k \leq[n / 2]$, where $[n / 2]$ stands for the integer part of $n / 2$. We shall prove that $M^{n}$ is compact. To this aim we distinguish two subcases.

Assume that $k=1$. Since $\sum_{i=1}^{n} \lambda_{i}=0$, we get $\lambda_{1} \geq n-1$. Obviously, we have $\tilde{K}\left(e_{i} \wedge e_{j}\right)>1$, if $i, j>1$. For the two plane spanned by $e_{1}$ and $e_{i}, i>1$, we have

$$
\tilde{K}\left(e_{1} \wedge e_{j}\right) \geq \frac{n-2}{n-1} .
$$

Therefore, all the sectional curvatures of $\left(M^{n},\langle\tilde{,}\rangle\right)$ are bounded below by a positive constant; so $M^{n}$ is compact by the Bonnet-Myers' theorem.

Assume that $k>1$. By virtue of $(3.3)$, the Ricci curvature of $\left(M^{n},\langle\tilde{\langle}\rangle\right)$ in the direction $e_{i}$ is given by

$$
\tilde{\operatorname{Ric}}\left(e_{i}\right)=n-1+\frac{1}{\lambda_{i}} \sum_{j \neq i} \frac{1}{\lambda_{j}} .
$$

Using (3.2), for $1 \leq i \leq k$, we have

$$
\tilde{\operatorname{Ric}}\left(e_{i}\right) \geq n-1+\frac{1}{\lambda_{i}} \sum_{j=k+1}^{n} \frac{1}{\lambda_{j}} \geq k-1>0 .
$$

Similarly, for $k+1 \leq i \leq n$, we find that $\tilde{\operatorname{Ric}}\left(e_{i}\right) \geq k-1>0$. Hence the Ricci curvature of $\left.\left(M^{n}, \tilde{\langle},\right\rangle\right)$ is bounded below by a positive constant. By the Bonnet-Myers' theorem, $M^{n}$ is compact. 
Moreover, (3.2) easily implies that $\left(M^{n},\langle\tilde{,}\rangle\right)$ has non-negative sectional curvatures. Denote by $\bar{M}^{n}$ the universal cover of $M^{n}$ with covering projection $\pi: \bar{M}^{n} \rightarrow M^{n}$. We consider the isometric minimal immersion $g=f \circ \pi: \bar{M}^{n} \rightarrow S^{n+1}$ and the immersion $\tilde{g}=\tilde{f} \circ \pi: \bar{M}^{n} \rightarrow S^{n+1}$, where $\tilde{f}=f_{\pi / 2}$. The above arguments applied to $\bar{M}^{n}$ and $g$ yield that $\bar{M}^{n}$ is compact with non-negative sectional curvatures and positive Ricci curvature with respect to the Riemannian metric induced on it by $\tilde{g}$. The proof of Theorem A(ii) in the case $n=3$ follows directly from the Hamilton's theorem [5] which states that a compact and connected Riemannian 3-manifold with positive Ricci curvature is diffeomorphic to a spherical space form. We treat the case where $n \geq 4$. Appealing to a result due to Baldin and Mercuri $\left[\mathbf{1}\right.$, Theorem 3.2] we infer that either $\bar{M}^{n}$ is a homotopy sphere or is isometric to a product $M_{1}^{d} \times M_{2}^{n-d}$ of two compact manifolds of non-negative curvature. Moreover, in the latter case there are isometric (convex) embeddings $f_{1}: M_{1}^{d} \rightarrow R^{d+1}$ and $f_{2}: M_{2}^{n-d} \rightarrow R^{n-d+1}$ such that $\tilde{g}=f_{1} \times f_{2}$. In the case where $\bar{M}^{n}$ is a homotopy sphere, by the generalized Poincare conjecture (Smale $n \geq 5$, Freedman $n=4$ ) we deduce that $\bar{M}^{n}$ is homeomorphic to $S^{n}$. The subspace spanned by the positive eigenspaces at each point defines a continuous $k$-plane field on $\bar{M}^{n}$. According to Steenrod [15], $n$ must be odd. In the case where $\bar{M}^{n}$ is isometric to a product and $\tilde{g}=f_{1} \times f_{2}$, we see that $\tilde{f}\left(M^{n}\right)$ is a product of two round hyperspheres. Hence $f\left(M^{n}\right)$ is also a product of two round hyperspheres. Since $f$ is minimal, we deduce that $f\left(M^{n}\right)=S^{m}(\sqrt{m / n}) \times S^{n-m}(\sqrt{(n-m) / n})$. Moreover, since supRic = $n-2$, we conclude that $n=2 m$ and $f\left(M^{n}\right)=S^{m}(\sqrt{1 / 2}) \times S^{m}(\sqrt{1 / 2})$. This completes the proof of Theorem A.

The main idea in the proof of Theorem B is to prove that $M^{n}$ is a homology sphere based on the following result due to Lawson and Simons [8].

Theorem 3.1. Let $M^{n}$ be a compact submanifold of the unit sphere $S^{n+k}$ with second fundamental form $B$. Let $p, q$ be positive integers such that $p+q=n$. If for any $P \in M^{n}$ and any orthonormal basis $\left\{e_{1}, \ldots, e_{p}, \ldots, e_{n}\right\}$ of $T_{P} M$, the following inequality is satisfied

$$
\sum_{i=1}^{p} \sum_{j=p+1}^{n}\left(2\left|B\left(e_{i}, e_{j}\right)\right|^{2}-\left\langle B\left(e_{i}, e_{i}\right), B\left(e_{j}, e_{j}\right)\right\rangle\right)<p q,
$$

then

$$
H_{p}\left(M^{n}, Z\right)=H_{q}\left(M^{n}, Z\right)=0,
$$

where $H_{i}\left(M^{n}, Z\right)$ denotes the $i$-th homology group of $M^{n}$ with integer coefficients.

Proof of Theorem B. If $n=3$, then it follows by a result due to Hamilton [5] that $M^{n}$ is diffeomorphic to a spherical space form. Hereafter, we 
assume that $n>3$. Let $P \in M^{n}$ and $\left\{e_{1}, \ldots, e_{p}, \ldots, e_{n}\right\}$ be an arbitrary orthonormal basis of $T_{P} M$. Since $M^{n}$ is minimal we have

$$
\begin{aligned}
& \sum_{i=1}^{p} \sum_{j=p+1}^{n}\left(2\left|B\left(e_{i}, e_{j}\right)\right|^{2}-\left\langle B\left(e_{i}, e_{i}\right), B\left(e_{j}, e_{j}\right)\right\rangle\right) \\
& =\sum_{i=1}^{p} \sum_{j=p+1}^{n} 2\left|B\left(e_{i}, e_{j}\right)\right|^{2}+\sum_{i=1}^{p} \sum_{i_{1}=1}^{p}\left\langle B\left(e_{i}, e_{i}\right), B\left(e_{i_{1}}, e_{i_{1}}\right)\right\rangle .
\end{aligned}
$$

We choose an orthonormal basis $\left\{e_{n+1}, \ldots, e_{n+k}\right\}$ of the normal space at $P$ and denote by $A_{n+1}, \ldots, A_{n+k}$ the corresponding shape operators. Then the above equality becomes

$$
\begin{aligned}
& \sum_{i=1}^{p} \sum_{j=p+1}^{n}\left(2\left|B\left(e_{i}, e_{j}\right)\right|^{2}-\left\langle B\left(e_{i}, e_{i}\right), B\left(e_{j}, e_{j}\right)\right\rangle\right) \\
& =2 \sum_{i=1}^{p} \sum_{j=p+1}^{n} \sum_{\alpha=n+1}^{n+k}\left\langle A_{\alpha} e_{i}, e_{j}\right\rangle^{2}+\sum_{\alpha=n+1}^{n+k}\left(\sum_{i=1}^{p}\left\langle A_{\alpha} e_{i}, e_{i}\right\rangle\right)^{2} .
\end{aligned}
$$

By Cauchy-Schwarz inequality we obtain

$$
\begin{aligned}
& \sum_{i=1}^{p} \sum_{j=p+1}^{n}\left(2\left|B\left(e_{i}, e_{j}\right)\right|^{2}-\left\langle B\left(e_{i}, e_{i}\right), B\left(e_{j}, e_{j}\right)\right\rangle\right) \\
& \leq 2 \sum_{i=1}^{p} \sum_{j=p+1}^{n} \sum_{\alpha=n+1}^{n+k}\left\langle A_{\alpha} e_{i}, e_{j}\right\rangle^{2}+p \sum_{\alpha=n+1}^{n+k} \sum_{i=1}^{p}\left\langle A_{\alpha} e_{i}, e_{i}\right\rangle^{2} .
\end{aligned}
$$

We suppose that $p \geq 2$. Then we get

$$
\begin{aligned}
& \sum_{i=1}^{p} \sum_{j=p+1}^{n}\left(2\left|B\left(e_{i}, e_{j}\right)\right|^{2}-\left\langle B\left(e_{i}, e_{i}\right), B\left(e_{j}, e_{j}\right)\right\rangle\right) \\
& \leq p \sum_{i=1}^{p} \sum_{j=p+1}^{n} \sum_{\alpha=n+1}^{n+k}\left\langle A_{\alpha} e_{i}, e_{j}\right\rangle^{2}+p \sum_{\alpha=n+1}^{n+k} \sum_{i=1}^{p}\left\langle A_{\alpha} e_{i}, e_{i}\right\rangle^{2} \\
& =p \sum_{i=1}^{p} \sum_{\alpha=n+1}^{n+k}\left(\sum_{j=p+1}^{n}\left\langle A_{\alpha} e_{i}, e_{j}\right\rangle^{2}+\left\langle A_{\alpha} e_{i}, e_{i}\right\rangle^{2}\right) .
\end{aligned}
$$

This implies that

$$
\sum_{i=1}^{p} \sum_{j=p+1}^{n}\left(2\left|B\left(e_{i}, e_{j}\right)\right|^{2}-\left\langle B\left(e_{i}, e_{i}\right), B\left(e_{j}, e_{j}\right)\right\rangle\right) \leq p \sum_{i=1}^{p} \sum_{\alpha=n+1}^{n+k}\left|A_{\alpha} e_{i}\right|^{2} .
$$


In view of (2.1), we get

$$
\sum_{i=1}^{p} \sum_{j=p+1}^{n}\left(2\left|B\left(e_{i}, e_{j}\right)\right|^{2}-\left\langle B\left(e_{i}, e_{i}\right), B\left(e_{j}, e_{j}\right)\right\rangle\right) \leq p \sum_{i=1}^{p}\left(n-1-\operatorname{Ric}\left(e_{i}\right)\right) .
$$

From our assumption on the Ricci curvature, we infer that

$$
\sum_{i=1}^{p} \sum_{j=p+1}^{n}\left(2\left|B\left(e_{i}, e_{j}\right)\right|^{2}-\left\langle B\left(e_{i}, e_{i}\right), B\left(e_{j}, e_{j}\right)\right\rangle\right)<p^{2} \frac{n+1}{n-1} .
$$

Obviously $p \neq q$. Without loss of generality we may assume that $p<q$, hence $n \geq 2 p+1$. Since $n \geq 2 p+1$, we finally have

$$
\sum_{i=1}^{p} \sum_{j=p+1}^{n}\left(2\left|B\left(e_{i}, e_{j}\right)\right|^{2}-\left\langle B\left(e_{i}, e_{i}\right), B\left(e_{j}, e_{j}\right)\right\rangle\right)<p^{2} \frac{n+1}{n-1} \leq p q .
$$

It follows from Theorem 3.1 that $H_{p}\left(M^{n}, Z\right)=H_{q}\left(M^{n}, Z\right)=0$ for all $2 \leq p, q \leq n-2$ and $p+q=n$. Since $H_{n-2}\left(M^{n}, Z\right)=0$, by the universal fundamental theorem $H^{n-1}\left(M^{n}, Z\right)$ has no torsion and consequently $H_{1}\left(M^{n}, Z\right)$ has no torsion by Poincare duality. By our assumption on the Ricci curvature and the Bonnet-Myers' theorem, we know that the fundamental group $\pi_{1}\left(M^{n}\right)$ of $M^{n}$ is finite. Hence $H_{1}\left(M^{n}, Z\right)=0$ and therefore by Poincare duality $H_{n-1}\left(M^{n}, Z\right)=0$. So $M^{n}$ is a homology sphere. The above arguments can be applied to the universal covering $\tilde{M}^{n}$ of $M^{n}$. Since $\tilde{M}^{n}$ is a homology sphere which is simply connected, i.e., $\pi_{1}\left(\tilde{M}^{n}\right)=0$, it is also a homotopy sphere. By the generalized Poincare conjecture (Smale $n \geq 5$, Freedman $n=4$ ) we have that $\tilde{M}^{n}$ is homeomorphic to $S^{n}$. A result due to Sjerve [12] implies that $\pi_{1}\left(M^{n}\right)=0$ and so $M^{n}$ is homeomorphic to $S^{n}$. This completes the proof of Theorem B.

\section{Concluding remarks and questions.}

(i). It is well known $[\mathbf{7}, \mathbf{1 6}]$ that for any $n \geq 3$ there is an abundance of minimal immersions or embeddings of the differentiable $n$-sphere into the standard Euclidean unit hypersphere $S^{n+1}$, which are not equators. By a similar argumentation as in the proof of Theorem A we obtain: If $n=2 m$, and $f: S^{n} \rightarrow S^{n+1}$ is a minimal immersion, then supRic $=n-1$. In fact, if supRic $<n-1$, then the principal curvatures $\lambda_{i}, i=1, \ldots, n$, of $f$ satisfy $\left|\lambda_{i}\right|>0$. So the subspace spanned by the positive eigenspaces at each point define a continuous $r$-plane field $(0<r<n)$, which is a contradiction by Steenrod [15]. It is natural to pose the following question: Let $f: S^{n} \rightarrow S^{n+1}$ be a minimal immersion of an odd-dimensional $n$-sphere. Is it true that $\operatorname{supRic}=n-1$ ? 
(ii). A direct computation shows that all odd $n$-dimensional Clifford minimal hypersurfaces satisfy

$$
\text { supRic } \geq \frac{n(n-1)}{n+1},
$$

with equality only in the case of $S^{m}(\sqrt{m / n}) \times S^{m+1}(\sqrt{(m+1) / n})$, where $n=2 m+1$. On the other hand, in [6] it has been proved that: Let $M^{3}$ be a compact and oriented 3-dimensional Riemannian manifold, and $f: M^{3} \rightarrow$ $S^{4}$ an isometric minimal immersion; then supRic $\geq 3 / 2$ and the equality holds only in the case where $f\left(M^{3}\right)=S^{1}(\sqrt{1 / 3}) \times S^{2}(\sqrt{2 / 3})$. Moreover, by an easy computation, we verify that the Cartan minimal hypersurfaces and the minimal hypersurfaces constructed by Otsuki in [11] do satisfy $(*)$. Taking all these as a clue, it is plausible to raise the following question. Let $M^{n}$ be a compact, oriented Riemannian manifold of dimension $n=2 m+1$ and $f: M^{n} \rightarrow S^{n+1}$ an isometric minimal immersion. Is it true that supRic $\geq n(n-1) /(n+1)$, with equality only in the case where $f\left(M^{n}\right)=$ $S^{m}(\sqrt{m / n}) \times S^{m+1}(\sqrt{(m+1) / n})$ ?

(iii). The following statement holds and its proof is elementary. Let $\lambda_{i}, i=$ $1, \ldots, n,(n=2 m+1)$ be real numbers satisfying $\sum_{i} \lambda_{i}=0$ and $\frac{m}{m+1} \leq \lambda_{i}^{2} \leq$ $\frac{m+1}{m}$; then $m$ of them (after an eventual change of sign) are equal to $\sqrt{\frac{m}{m+1}}$ and the remainder are equal to $-\sqrt{\frac{m+1}{m}}$. Moreover, $\sum_{i} \lambda_{i}^{2}=n$. In fact, without loss of generality, we may suppose that the numbers $\lambda_{1}, \ldots, \lambda_{k},(1 \leq$ $k \leq m)$ are positive and the numbers $\lambda_{k+1}, \ldots, \lambda_{n}$ are negative. Since $\lambda_{1}+\cdots+\lambda_{k}=-\lambda_{k+1}-\cdots-\lambda_{n}$, we obtain $(n-k) \sqrt{\frac{m}{m+1}} \leq k \sqrt{\frac{m+1}{m}}$, or equivalently $k \geq m$. So $k=m$. Now from $-\sqrt{\frac{m+1}{m}} \leq \lambda_{2 m+1} \leq-\sqrt{\frac{m}{m+1}}$ and $\lambda_{2 m+1}=-\sum_{i=1}^{2 m} \lambda_{i}$, we obtain

$$
\sqrt{\frac{m+1}{m}} \geq \sum_{i=1}^{2 m} \lambda_{i} \geq \sqrt{\frac{m}{m+1}} .
$$

On the other hand, we have

$$
\sum_{i=1}^{2 m} \lambda_{i} \leq m \sqrt{\frac{m+1}{m}}-m \sqrt{\frac{m}{m+1}}=\sqrt{\frac{m}{m+1}},
$$

which implies the desired result.

As an application of the above observation, we get the following local result: Let $M^{n}$ be an odd-dimensional Riemannian manifold and $f: M^{n} \rightarrow$ $S^{n+1}$ an isometric minimal immersion satisfying $\frac{n(n-3)}{n-1} \leq \operatorname{Ric} \leq \frac{n(n-1)}{(n+1)}$; 
then $f\left(M^{n}\right)$ is an open portion of $S^{m}(\sqrt{m / n}) \times S^{m+1}(\sqrt{(m+1) / n})$. In fact, by the hypothesis on the Ricci curvature, the principal curvatures $\lambda_{i}, i=$ $1, \ldots, n$, satisfy $\frac{m}{m+1} \leq \lambda_{i}^{2} \leq \frac{m+1}{m}$ at every point. So the square length $S$ of the second fundamental form satisfies $S=n$ and the deduction is standard.

Remark. This result has been proved by Haizhong [4] for $n=3$ under the global assumption of compactness.

(iv). In [10, p. 389] Leung posed the following conjecture: Let $n=2 m+1$. Suppose that $M^{n}$ is a compact and connected immersed minimal submanifold of the unit sphere $S^{n+k}$. If $|B(X, X)|^{2}<\frac{n+1}{n-1}$ for any unit tangent vector $X$ at any point, where $B$ is the second fundamental form, then $M^{n}$ is homeomorphic to $S^{n}$. Theorem B provides a partial confirmation of this conjecture, since our assumption Ric $>n(n-3) /(n-1)$ implies that $|B(X, X)|^{2}<\frac{n+1}{n-1}$ for any unit tangent vector $X$. However, in the case where the normal connection of $M^{n}$ is flat, the condition $|B(X, X)|^{2}<\frac{n+1}{n-1}$ is equivalent to Ric $>n(n-3) /(n-1)$. So under this assumption, Theorem $\mathrm{B}$ proves the above conjecture.

\section{References}

[1] Y. Baldin and F. Mercuri, Isometric immersions in codimension two with nonnegative curvature, Math. Z., 173 (1980), 111-117.

[2] M.P. do Carmo and N.R. Wallach, Minimal immersions of spheres into spheres, Annals of Math., 93 (1971), 43-62.

[3] M.P. do Carmo and F.W. Warner, Rigidity and convexity of hypersurfaces in spheres, J. Diff. Geom., 4 (1970), 133-144.

[4] L. Haizhong, A characterization of Clifford minimal hypersurfaces in $S^{4}$, Proc. Amer. Math. Soc., 123 (1995), 3183-3187.

[5] R.S. Hamilton, Three-manifolds with positive Ricci curvature, J. Diff. Geom., 17 (1982), 255-306.

[6] Th. Hasanis and D. Koutroufiotis, Applications of the Gauss mapping for hypersurfaces of the sphere, Lecture Notes in Math., Vol. 1156, Springer-Verlag, 180-193.

[7] W.Y. Hsiang and I. Sterling, Minimal cones and the spherical Bernstein problem III, Invent. Math., 85 (1986), 223-247.

[8] H.B. Lawson and J. Simons, On stable currents and their application to global problems in real and complex geometry, Annals of Math., 98 (1973), 427-450.

[9] P.F. Leung, Minimal submanifolds in a sphere, Math. Z., 183 (1983), 75-86.

[10] _ Minimal submanifolds in a sphere II, Bull. London Math. Soc., 23 (1991), 387-390.

[11] T. Otsuki, Minimal hypersurfaces in a Riemannian manifold of constant curvature, Amer. J. Math., 92 (1970), 145-173.

[12] D. Sjerve, Homology spheres which are covered by spheres, J. London Math. Soc., 6 (1973), 333-336. 
[13] B. Smyth, Efimov's inequality and other inequalities in a sphere, Geometry and Topology of Submanifolds, IV; 76-86, World Scientific, 1991.

[14] - The principal curvature set of a hypersurface in a sphere, Geometry and Topology of Submanifolds, V; 260-266, World Scientific, 1992.

[15] N. Steenrod, The Topology of Fibre Bundles, Princeton University Press, Princeton, New Jersey, 1951.

[16] P. Tomter, The spherical Bernstein problem in even dimensions and related problems, Acta Math., 158 (1987), 189-212.

Received April 29, 1999.

UNIVERSITY OF IOANNINA

IOANNINA 45110

Greece

E-mail address: thasanis@cc.uoi.gr

UNIVERSITY OF IOANNINA

IOANNINA 45110

GREECE

E-mail address: tvlachos@cc.uoi.gr 